\title{
Desenvolvimento da Superfície Livre em Escoamentos Aerados: Analogia com Leis Básicas de Transferência
}

\author{
Harry Edmar Schulz ${ }^{1}$, André Luiz Andrade Simões ${ }^{2}$ \\ heschulz@sc.usp.br, andre.simoes@pro.unifacs.br
}

Recebido: 22/08/11 - revisado: 21/12/11 - aceito: 19/06/13

\begin{abstract}
RESUMO
A interface em escoamentos bifásicos turbulentos pode ser de difícil definição, eventualmente gerando dificuldades de quantificação das propriedades relevantes. No caso de escoamentos de ar e água, a grande diferença de densidades faz com que, em muitas aplicações práticas, o sistema esteja estratificado, com maior porcentagem de água na região inferior e maior porcentagem de ar na região superior. Adicionalmente, para dimensões naturais, a atmosfera pode ser considerada isenta de água e os escoamentos profundos podem ser considerados isentos de ar. Nessas condições, pode-se estudar mais objetivamente a posição das interfaces superior e inferior da mistura ar-água, sendo que o presente estudo fixa-se na posição da interface superior, isto é, no seu contato com a atmosfera. $\mathrm{O}$ uso das leis físicas de conservação sempre conduz a equacionamentos úteis, mas, não raro, implicam em numerosos ajustes para levar em conta os detalhes simplificados. Neste estudo fornece-se uma formulação considerando a observação do fenômeno e suas variáveis interferentes imediatas, procedimento comum na área de Fenômenos de Transporte, produzindo conclusões fisicamente coerentes. A formulação é aplicada no cálculo do comprimento entre zona não aerada e zona completamente aerada em canais retangulares escalonados.
\end{abstract}

Palavras-Chave: Fenômenos de transporte. Escoamentos bifásicos. Canal em degraus.

\section{INTRODUÇÃO}

Ao trabalhar com escoamentos multifásicos, o pesquisador muitas vezes se vê às voltas com a necessidade de limitar geometricamente o escoamento, ou, em outras palavras, delimitar uma posição média na qual suas observações devem ser consideradas. Tal situação se verifica no estudo de escoamentos de água em grandes velocidades e com superfície livre. Em algumas situações, a característica de quebra da superfície, com enlaçamento de bolhas de ar e geração de gotas, é considerada benéfica para as utilizações do escoamento. Esse é o caso da aeração em estruturas hidráulicas potencialmente sujeitas à cavitação. A Escola de Engenharia de São Carlos, Universidade de São Paulo, vem desenvolvendo sequencialmente estudos em escoamentos

\footnotetext{
1 - Departamento de Engenharia Hidráulica e Saneamento EESC/USP

${ }^{2}$ - Universidade Salvador - UNIFACS
}

aerados de alta velocidade, podendo ser mencionados os estudos básicos de Carvalho (1997) e Lima (2003); o primeiro tendo construído um canal com declividade variável, e o segundo tendo implementado nestes equipamentos de medições precisas, como um sistema de velocimetria a laser e um sistema de obtenção de concentrações de bolhas a partir de radiação de baixa potência, com o qual um banco de dados valioso foi elaborado. Seus estudos envolveram a medida da aeração através de aeradores de fundo e o registro da aeração superficial. Parte desses dados foi utilizada em estudos quantitativos nos quais se buscou avançar na obtenção das relações básicas para a aeração, fundamentadas em princípios físicos. Nesse sentido, podem ser mencionados os estudos de Brito (2011) e Lima, Schulz e Gulliver (2008). Os escoamentos estudados por esses autores foram majoritariamente para canais lisos, sendo que Carvalho (1997) ainda apresentou dados para rampas rugosas nos aeradores de fundo. Schulz (2008) realizou um estudo que envolveu diferentes aspectos de escoamentos bifásicos, um dos quais envolvendo aeradores de fundo e outro envolvendo vertedores escalonados e sua aeração (ver também LUEKER et al., 2008 - Folsom Dam). 
Em ambos os aspectos mencionados, buscou-se quantificar a aeração convenientemente. Em termos de simulação computacional, Arantes (2007) buscou reproduzir os experimentos de Lima (2003) para canais lisos, bem como os experimentos de diversos autores nacionais para canais escalonados, nesse último caso voltando-se ao problema da aeração e da proposição de um aerador de fundo a ser adotado em vertedores lisos ou escalonados. Simões (2008), reunindo dados gerados para vertedores escalonados em diferentes centros de estudo do país e do exterior, apresentou análises dimensionais muito convincentes, que permitiram observar tendências comuns aos diferentes dados, e forneceu um equacionamento para a quantificação do comprimento de bacias de dissipação, estudo que foi posteriormente complementado para também quantificar o fator de resistência nos vertedores, conforme pode ser visto em Simões, Schulz e Porto (2010).

Descrições relativas aos escoamentos em vertedores lisos possuem alguns aspectos em comum com aquelas apresentadas para estruturas em degraus. A região inicial do escoamento é composta apenas por água (Região 1 - Figura 1), com uma superfície livre aparentemente lisa. A partir da posição onde a espessura da camada limite coincide com a profundidade do escoamento, define-se o ponto de início da aeração (ver Figura 1). É nesta posição que os efeitos originados no fundo podem ser percebidos na superfície, tornando-a bastante irregular e contorcida. A jusante do início da aeração, desenvolve-se um campo de fração volumétrica de ar $C\left(x_{i}, t\right)$ cuja evolução espacial em $x_{1}$ (coordenada longitudinal) é crescente desde a superfície até o fundo, como ilustrado na figura 1a,b.

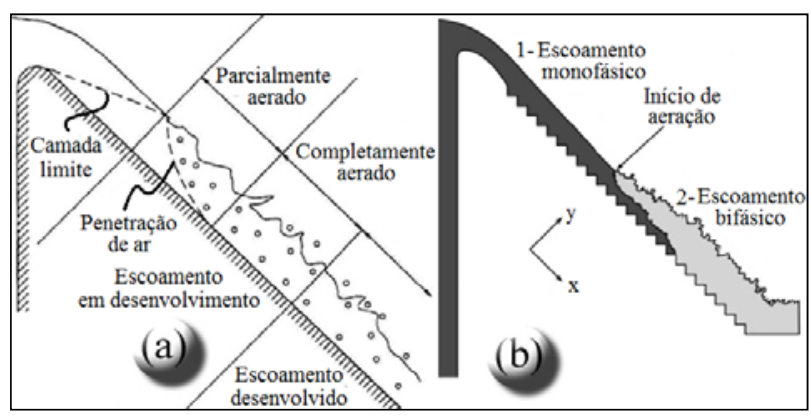

Figura 1 - (a) Escoamento em vertedor liso com ponto de início da aeração e suas regiões. (b) Escoamento deslizante sobre turbilhões com regiões

Fonte: (a) adaptado de Keller, Lai e Wood (1974);

(b) Simões (2011)
A informação existente sobre escoamento em canais lisos indica que a região (1) é monofásica. $\mathrm{O}$ mesmo ocorre em vertedores em degraus de barragens e modelos físicos utilizados em laboratórios. Entretanto, canais curtos com entradas laterais ou em queda livre, como aqueles existentes em sistemas de drenagem, normalmente operam com escoamentos aerados desde a posição inicial. Iniciando a aeração, há a evolução espacial dos perfis de fração de vazios, isto é, para valores médios (temporais) de $C$, denotados por $\bar{C}$, há uma função $\bar{C}(x, y)$, adotando a hipótese de escoamento bidimensional. A partir de uma determinada posição $x$, o equilíbrio é estabelecido de tal maneira que $\bar{C}=\bar{C}(y)$. Trabalhos como os de Cain e Wood (1981), Keller, Lai e Wood (1974), Straub e Anderson (1958) e Wood, Ackers e Loveless (1983) apresentaram resultados coerentes com essas descrições, atrelados aos escoamentos em vertedores lisos. A figura 1b é um exemplo clássico, apresentado por Keller, Lai e Wood (1974).

A quantidade de ar presente na mistura arágua é de grande importância, pois frações de vazios entre 5 e $8 \%$ são suficientes para evitar danos decorrentes da cavitação (PETERKA, 1953). Nas proximidades do início da aeração superficial não há uma quantidade de bolhas suficiente para evitar a cavitação junto ao fundo do canal. Embora ocorram movimentos de grandes grupos de bolhas em direção ao fundo, em média, observa-se o desenvolvimento descrito anteriormente para a fração de vazios.

Simões (2011) mediu a posição média da superfície livre com um sensor acústico ultrassônico, com taxa de amostragem de $50 \mathrm{~Hz}$ durante $120 \mathrm{~s}$ e ao longo de 40 posições em um canal em degraus com 3,5 m de comprimento útil. A região cônica formada pelas ondas acústicas possuía ângulo de abertura em torno de $7^{\circ}$ e a resolução do equipamento foi de $1 \mathrm{~mm}$. Para a região a montante do início da aeração superficial, os resultados apontaram a existência de um perfil da superfície livre caracterizado por profundidades decrescentes no sentido do escoamento. Empregando a terminologia de Chow (1959), essa forma é denominada como $S_{2}$, curva pertencente à classe $\mathrm{S}$ que ocorre em canais de declividade forte. Entre o início da aeração e o escoamento plenamente bifásico foi identificado um trecho de transição. Note-se que essa transição não é convenientemente quantificada com os modelos de superfície livre atualmente existentes. $O$ presente estudo permite, entre outras conclusões, justificar o comportamento da superfície livre e apresentar 
modelos matemáticos para o comprimento de transição mencionado.

\section{Modelo físico-matemático proposto para o comprimento de transição}

Considere-se o escoamento de transição indicado na figura 2, e o volume de controle a ele associado. O desenho esquemático apresentado na figura $2 \mathrm{a}$ e a fotografia da figura $2 \mathrm{~b}$ ilustram a forma contorcida da superfície livre a jusante do ponto de início da aeração. A figura 2c corresponde a um perfil médio obtido com um sensor acústico.

O presente estudo parte da assim denominada taxa de geração de vazios entre as seções 1 e 2 , indicada pela letra $\dot{C}$. Para a construção do modelo proposto, a observação permite sugerir que essa taxa é proporcional à vazão específica que atravessa o volume indicado na figura 2.

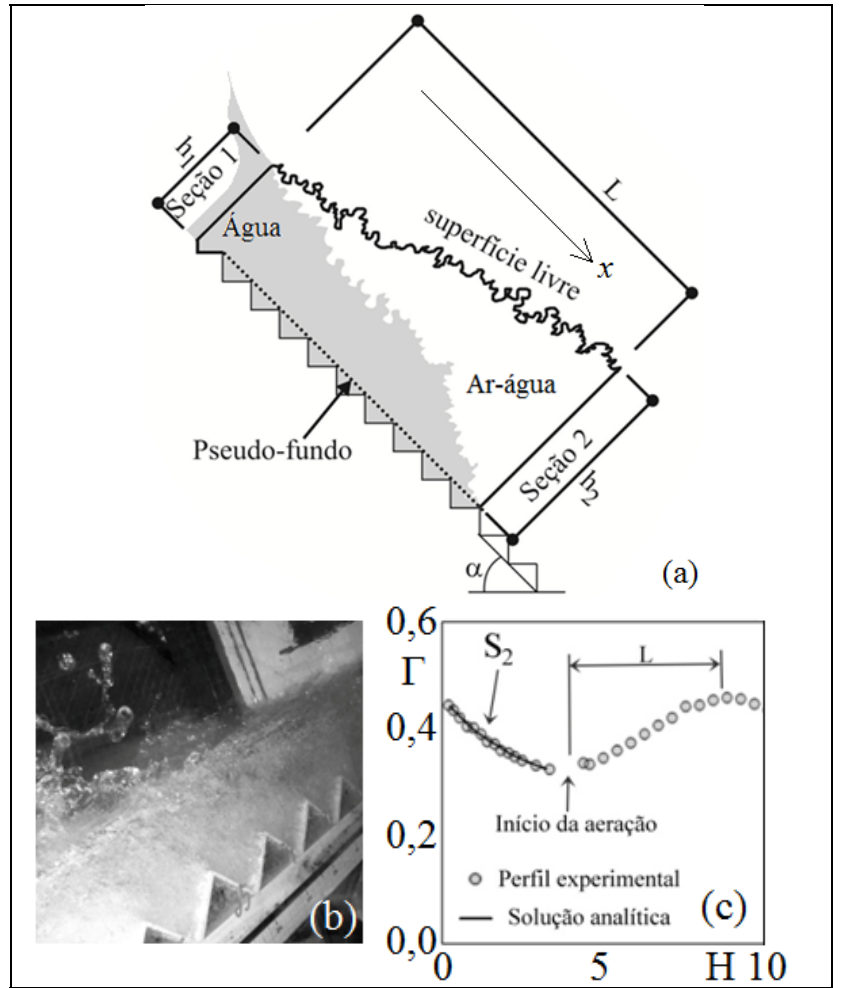

Figura 2 - (a) Escoamento bifásico e comprimento de transição (L) em canais escalonados; (b) Superfície livre do escoamento bifásico e (c) Perfil médio da superfície

livre, em que $\Gamma=h / h_{c}$ e $H=z / h_{c}(h=$ altura do escoamento perpendicular ao fundo, $h_{c}=$ profundidade crítica, $z=$ eixo vertical com origem na extremidade inicial do canal) Fonte: Simões (2011), figuras b e c.
A proporcionalidade pode também envolver uma potência desta vazão, mas a abordagem mais simples (primeira potência) é aqui considerada. Adicionalmente, quanto maior a inclinação positiva da interface entre a "água branca" (mistura de vazios e água) e a atmosfera, mais intensa é a geração de vazios no trecho. Assim, $\dot{C}$ também é apresentado como proporcional à inclinação da posição média da superfície livre. Eventualmente também aqui a proporcionalidade pode envolver potências da declividade, mas novamente a abordagem mais simples é adotada. Matematicamente, escreve-se:

$\left\{\begin{array}{l}\dot{C} \propto q \\ \dot{C} \propto \frac{d h}{d x}\end{array}\right.$

em que $q=$ vazão específica, $h=$ altura de escoamento, $x=$ coordenada longitudinal (ver Figura 2).

Como decorrência imediata das proporcionalidades presentes na equação 1 , pode-se definir a seguinte equação:

$\dot{C}=K q \frac{d h}{d x}$

Na equação 2 a vazão específica de água é constante em todo o volume e é uma característica de cada experimento. Entretanto, a taxa de geração de vazios é uma função da posição ao longo de $x$. Isto pode ser visualizado também na figura 1 . Antes de atingir o volume no qual os vazios são gerados, tem-se "água preta", ou seja, uma situação idealmente contendo apenas água. Assim, o contorno de montante pode ser indicado como $\dot{C}=0$. Da mesma maneira, a jusante do volume, a razão de vazios na água mantém-se idealmente constante, indicando que novamente $\dot{C}=0$. Assim, $\dot{C}$ evolui de valores baixos nos contornos de montante e jusante, e atinge valores máximos dentro do volume.

O fator $K$, a exemplo das "constantes de proporcionalidade" das leis básicas de transferência de massa e calor (leis de Fick e Fourier), pode variar de acordo com as características do meio, bem como eventualmente ser uma função da própria variável dependente. No caso da lei de Fick, a difusividade molecular depende dos compostos que difundem e do meio no qual isso ocorre. Adicionalmente, essa difusividade pode ser função da concentração de massa na qual se está trabalhando. As mesmas observações valem para a lei de Fourier e a condutividade térmica: esta depende do material que conduz o 
calor e pode ser dependente da temperatura. Mais equações análogas, como a lei da viscosidade de Newton e a lei de Darcy para meios porosos, também podem ser evocadas, com suas "constantes de proporcionalidade": viscosidade dinâmica e condutividade hidráulica. Uma apresentação bastante didática dessas leis básicas pode ser encontrada em Schulz (2003). Uma aplicação da equação 2 quantificando a forma da superfície multifásica encontrase em Schulz, Lobosco e Simões et al. (2011).

No presente estudo, o parâmetro que caracteriza o meio é a vazão específica. Entretanto, também as condições nas quais o fluido se desloca são importantes. A turbulência intrínseca ao movimento, causada ou inibida por condições de rugosidade, excitação prévia (a montante), viscosidade, podem entrar na composição desta constante.

Como é usualmente feito nos estudos de Fenômenos de Transporte, uma primeira abordagem considerando $\dot{C}$ e $K$ constantes é apresentada. Separando as variáveis e integrando, resulta:

$$
\dot{C} \int_{0}^{L} d x=K q \int_{h_{1}}^{h_{2}} d h \Rightarrow L=\frac{K q}{\dot{C}}\left(h_{2}-h_{1}\right)
$$

$K$ pode, como comentado, ser variável. Deste modo, para permitir uma generalização, considerou-se uma aproximação por meio de uma série do tipo:

$$
K=\sum_{i} \omega_{i}(q h)^{i}
$$

$\omega_{i}$ representa coeficientes de ajuste. Substituindo a equação 4 na equação 3 e usando truncamento de primeira ordem $(i=1)$, obtém-se:

$$
\begin{aligned}
& \dot{C}=\sum_{i} \omega_{i}(q h)^{i} \frac{d q h}{d x} \Rightarrow \dot{C} d x=\left(\omega_{0}+\omega_{1} q h\right) d(q h) \Rightarrow \\
& \dot{C} L=\omega_{0}\left(q h_{2}-q h_{1}\right)+\frac{\omega_{1}}{2}\left(q^{2} h_{2}^{2}-q^{2} h_{1}^{2}\right) \\
& L=\frac{\omega_{0}}{\dot{C}}\left(q h_{2}-q h_{1}\right)+\frac{\omega_{1}}{2 \dot{C}}\left(q^{2} h_{2}^{2}-q^{2} h_{1}^{2}\right)
\end{aligned}
$$

Evidentemente pode-se estender essa aproximação para ordens superiores $(i=2,3$, etc.), mas o presente estágio dos estudos não exige esse procedimento. Um aspecto positivo de possuir uma nova formulação é que novas formas de dependência entre os parâmetros que descrevem o fenômeno ficam aparentes. As equações 2 e 5, por exemplo, sugerem que $L$ deva ser dependente dos produtos $q h_{1}$ e $q h_{2}$. Seguindo a mesma forma da equação 5, uma expressão semi-empírica pode então ser sugerida, como:

$$
L=\theta_{1} q h_{2}+\theta_{2} q h_{1}+\theta_{3}\left(q h_{2}\right)^{2}+\theta_{4}\left(q h_{1}\right)^{2}+\theta_{5}
$$

A equação 6 evidentemente deve ter os coeficientes $\theta_{1}$ a $\theta_{5}$ ajustados a partir de resultados experimentais.

\section{ASPECTOS FÍSICOS DOS PARÂMETROS ENVOLVIDOS}

Considerando a dependência de $\dot{C}$ para $\operatorname{com} x$, é interessante observar os seguintes pontos:

- $\quad \dot{C}$ possui uma região de máximo no volume, o que faz com que a aproximação mais simples para $\dot{C}$ seja uma equação de segunda ordem em $x$ (ou, em outros termos, um polinômio de grau 2).

- Sendo assim, considerando o caso mais simples em que $K$ é uma constante, isso implica que a evolução da superfície livre é nãolinear, sendo regida no mínimo por uma função de terceira ordem em $x$ (ou, em outros termos, um polinômio de grau 3). Essa condição é fisicamente necessária, porque a superfície evolui nessa região (volume) como uma curva em S, e apenas com funções de terceira ordem ou superiores pode-se gerar um ponto de inflexão. Essa correlação direta entre $\dot{C}$ e $h$ é imediatamente observável pela presente formulação. Adicionalmente, se $K$ variar, sabe-se que $\dot{C}$ seguirá um comportamento que imporá no mínimo uma função de terceira ordem para $h$.

- Qualquer função que se utilize para representar $\dot{C}$ como função de $x$ não afeta a generalidade da equação 2. Para demonstrar isto, considere-se o desenvolvimento a seguir.

A função genérica envolve $x$ multiplicado por alguma constante a determinar. É possível então adimensionalizar esta função na forma: 
$\dot{C}=\dot{C}\left(a_{i} x\right) \Rightarrow \dot{C}=\dot{C}\left(a_{i} L \frac{x}{L}\right) \Rightarrow$

$\dot{C}=\dot{C}\left(b_{i} \frac{x}{L}\right)$

$a_{i}$ representa a(s) constantes(s) a determinar. As equações 2 e 7 permitem escrever:

$$
\dot{C}\left(b_{i} \frac{x}{L}\right) d\left(\frac{x}{L}\right)=\frac{K q}{L} d h
$$

$b_{i}$ representa a(s) novas constantes(s) a determinar. A integração do primeiro membro da equação 8 é feita entre 0 e 1 , fornecendo, portanto, um valor numérico, denotado aqui por $\psi$. O comprimento $L$, portanto, fica determinado por:

$$
L=\frac{1}{\psi} \int_{h_{1}}^{h_{2}} K q d h
$$

\section{POSSÍVEIS ADIMENSIONALIZACÕES EM BUSCA DA GENERALIZAÇÃO}

\section{Adimensionalização baseada no comprimento máximo (L)}

A equação 8 envolve a razão adimensional $x / L$, o que sugere que se busque formas adimensionais que permitam generalizar os resultados a serem obtidos. A primeira adimensionalização, sugerida diretamente da equação 8, é:

$$
\frac{\dot{C}}{K q}=\frac{d(h / L)}{d(x / L)} \Rightarrow C^{*}=\frac{d h^{*}}{d x^{*}}
$$

em que $C^{*}=$ taxa de incorporação de ar adimensional. Os asteriscos representam grandezas adimensionais, indistintamente, no presente estudo.

\section{Adimensionalização baseada em forças de gravidade $e$} viscosas

O escoamento em canais inclinados é impulsionado pelo peso (decorrente da força gravitacional), podendo ser impulsionado ou freado por forças de pressão (igualmente decorrentes da força gravitacional) e é freado por forças viscosas. A força gravitacional é geralmente representada pela aceleração da gravidade, $g$, enquanto que a força viscosa é geralmente representada pela viscosidade cinemática, $v$. As equações 3 e 5 mostram que $L$ depende de $q h$ e $\dot{C}$. Assim, uma análise dimensional que considere as forças mencionadas envolve os parâmetros $L, \dot{C}, q h, g$, v. Os cinco parâmetros dependem apenas das dimensões "comprimento" (m) e "tempo" (t). Portanto, três adimensionais independentes podem ser definidos, tendo sido obtidos os seguintes (indicados por $\pi_{i}$ ):

$\pi_{1}=L g^{1 / 3} v^{-2 / 3}=L^{*}$,

$\pi_{2}=\dot{C} g^{-2 / 3} v^{-1 / 3}=C^{*}$,

$\pi_{3}=q h g^{1 / 3} v^{-5 / 3}=h^{*}$.

Segue novamente, dessa análise dimensional, que $C^{*} \propto \frac{d h^{*}}{d x^{*}}$, sendo que a constante de proporcionalidade agora é um número puro e, de forma geral, não há qualquer modificação relativa às conclusões anteriores, porque as grandezas utilizadas para a adimensionalização são constantes físicas (invariantes).

\section{Adimensionalização baseada na geometria dos degraus}

Pode-se também propor uma adimensionalização em que as grandezas geométricas relacionadas às dimensões dos degraus sejam consideradas. Isso pode ser feito a partir do uso da definição do número de Froude em termos da altura de rugosidade $k=s \cos \alpha$, em que $s=$ altura do degrau e $\alpha$ é o ângulo formado entre o pseudo fundo e a horizontal, como ilustrado na figura 2. Pretende-se, ao incluir $k$ na formulação, considerar a influência da dimensão característica dos grandes turbilhões formados entre degraus e, portanto, de um fenômeno que ocorre junto ao fundo. $\mathrm{O}$ efeito dessa rugosidade sobre a taxa de geração de vazios não é tão claramente perceptível quanto o da própria vazão específica. Pode-se esperar que quanto mais rugoso o fundo, mais intensa seria a turbulência e, em princípio, maior seria a taxa de geração de vazios. Isso eventualmente é verdade para os menores valores de $s$ (ou $k$ ) em relação à profundidade média (até o pseudo fundo), mas não necessariamente se verifica para grandes valores de $s$ (ou $k$ ). Isso porque a energia do escoamento médio, que desliza sobre o pseudo fundo, é transferida mais intensamente para o movimento dos turbilhões entre grandes degraus, ao invés de gerar turbulência no próprio escoamento médio. Assim, esse movimento bem ordenado, em grandes degraus, atua permitindo o deslizamento 
com menor geração de turbulência junto ao pseudo fundo. Não obstante essa tendência teórica divergente para os maiores e menores degraus, $k$ pode ser usado como um parâmetro de adimensionalização, como qualquer comprimento característico, sendo que esse procedimento deve ser testado a partir de diferentes situações de rugosidade. Com essa adimensionalização, a taxa de incorporação de ar pode ser adimensionalizada com a vazão específica e o quadrado de $k$, como escrito a seguir:

$$
\left\{\begin{array}{l}
C^{*} \propto \mathrm{Fr}^{*}, \text { sendo } C^{*}=\dot{C} k^{2} / q, \\
\mathrm{Fr}^{*}=q / \sqrt{g k^{3} \operatorname{sen} \alpha} \\
C^{*} \propto \frac{d h}{d x}
\end{array}\right.
$$

Note-se que esta forma de abordagem não produz imediatamente a proporcionalidade expressa pela equação 2, mas uma proporcionalidade entre $\dot{C}$ e o quadrado de $q$, uma situação possível, conforme mencionado no parágrafo precedente à equação 1 . Entretanto, como a constante $K$ pode ser expressa como função da vazão (equação 4), essa diferença é suprimida. As variáveis $q, k, g, d h / d x$ e $\dot{C}$ produzem os adimensionais da equação 11 usando o teorema de Vaschy-Buckingham, exceto, evidentemente, pela presença do sen $(\alpha)$ que já é um adimensional. A adoção dessa definição do número de Froude está sendo testada devido ao seu uso frequente na representação matemática da posição de início da aeração e da profundidade no início da aeração.

Como consequência da equação 11, utilizando o coeficiente de proporcionalidade $\varphi$, (adimensional), tem-se:

$C^{*}=\varphi F^{*} \frac{d h}{d x}$

Separando as variáveis, integrando para $C^{*}$, $F r^{*}$ e $\varphi$ constantes, e definindo os adimensionais $L^{*}$ $=L / h_{1}$ e $h^{*}=h_{2} / h_{1}$, obtém-se:

$L^{*}=\frac{\varphi}{C^{*}} \operatorname{Fr}^{*}\left(h^{*}-1\right)$

$\mathrm{Ou}$

$$
\left\{\begin{array}{l}
L^{*}=\varphi_{1} \frac{q}{k^{2}} \operatorname{Fr}^{*}\left(h^{*}-1\right) \\
\varphi_{1}=\frac{\varphi}{\dot{C}}
\end{array}\right.
$$

A escolha dos adimensionais $L^{*} \mathrm{e} h *$ é conveniente, porque se referem à dimensão $h_{1}$, profundidade que pode ser calculada através de equacionamentos conhecidos, como aqueles apresentados em Simões, Schulz e Porto (2010). Adicionalmente, já se verificou sua adequação às formulações fundamentadas nos princípios de conservação de massa, quantidade de movimento e energia, como apresentado em Simões (2011).

Na equação 4 admitiu-se que $K$ pode ser uma variável, adotando-se uma forma de série e integrando a equação decorrente desta série. Essa integração foi necessária porque foi admitido que $K$ pode variar com a profundidade do escoamento. $\mathrm{Na}$ presente aproximação admitiu-se que a constante é de fato invariável para com as grandezas de integração $(x$ e $h)$, podendo ser uma função apenas da vazão específica e da rugosidade, o que é expresso pelo número de Froude. Adotando essa aproximação, devido à simplicidade da relação $13 \mathrm{~b}$, consideraram-se duas séries:

$\varphi_{1}=\sum_{i=0}^{\infty} a_{i} \operatorname{Fr}^{* b_{i}}=a_{0} \operatorname{Fr}^{* b_{0}}+a_{1} \operatorname{Fr}^{* b_{1}}+\ldots$

$\varphi_{1}=\sum_{i=0}^{\infty} a_{i} F r^{* i}=a_{0}+a_{1} F r^{*}+a_{2} F r^{* 2}+\ldots$

Substituindo os termos obtidos pelo truncamento para $i=1$ (equação 14) na equação $13 \mathrm{~b}$, obtém-se:

$L^{*}=\frac{q}{k^{2}}\left(h^{*}-1\right)\left[a_{0} F r^{* b_{0}+1}+a_{1} F r^{* b_{1}+1}\right]$

Substituindo a equação 15 na equação $13 \mathrm{~b}$, obtém-se: 
$L^{*}=\frac{q}{k^{2}}\left(h^{*}-1\right)\left(a_{0} F r^{*}+a_{1} F r^{* 2}+a_{2} F r^{* 3}\right)$

Segunda adimensionalização baseada na geometria dos degraus $(k)$

Considerando as grandezas $L$ e $h_{i}(i=1,2)$ na análise dimensional mencionada após a equação 11 , obtém-se, adicionalmente, $L / k$ e $h_{i} / k$. Isso sugere também que a integração da equação 12 pode ser posteriormente adimensionalizada dividindo-se ambos os membros por $k$ (ao invés de $h_{1}$ ), como mostrado na equação 18. Também nessa equação podem ser adotadas correções para $\varphi_{1}$, como as séries 14 e 15.

$\frac{L}{k}=\varphi_{1} \frac{q}{k^{2}} F^{*}\left(\frac{h_{2}-h_{1}}{k}\right)$

\section{CÁLCULO DAS CONSTANTES}

\section{Equações 5 e 6}

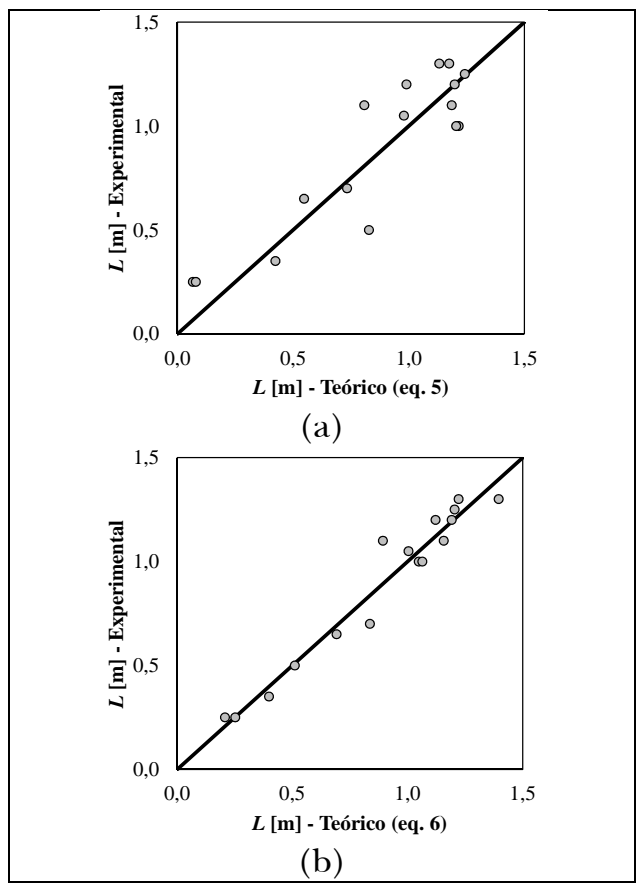

Figura 3 - Comparação entre valores medidos e calculados do comprimento de transição " $L$ ": (a) Valores calculados com a equação 5; (b) Valores calculados com a equação 6
As constantes presentes nas equações 5 e 6 foram calculadas minimizando-se a soma dos erros quadráticos entre valores experimentais e teóricos de L. Os resultados obtidos foram: 1) Equação 5: $\omega_{0} / \dot{C}=290,7, \omega_{1} /(2 \dot{C})=-2290,1$ e coeficiente de correlação igual a 0.90; 2) Equação 6: $\theta_{1}=789,12, \theta_{2}$ $=-976,5, \theta_{3}=-10610,5, \theta_{4}=17265,15, \theta_{5}=0,192 \mathrm{e}$ coeficiente de correlação igual a 0,98 . As figuras 3a e $3 \mathrm{~b}$ comparam os resultados experimentais com os valores teóricos do comprimento de transição calculados com as equações 5 e 6 .

Equação $13 b \operatorname{com} \varphi_{1}\left(\mathrm{Fr}^{*}\right)$

Se $\varphi_{1}$ for considerado constante, não há boa correlação entre valores medidos e calculados para $L^{*}$. Como primeira aproximação, calcularam-se valores de $\varphi_{1}$ para cada $\mathrm{Fr}^{*}$. Daí estabeleceu-se uma função de ajuste $\varphi_{1}\left(F r^{*}\right)$ com $\mathrm{R}^{2}=0,97$, como ilustrado na figura 4 a. Essa função foi substituída na equação $13 \mathrm{~b}$ e os valores de $L^{*}$ foram recalculados. Os resultados obtidos podem ser vistos na figura $4 \mathrm{~b}$ em comparação aos experimentais. O coeficiente de correlação para esse ajuste resultou igual a 0,87 .

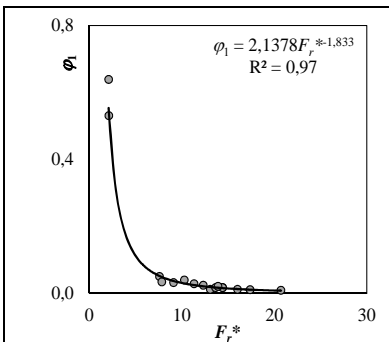

(a)

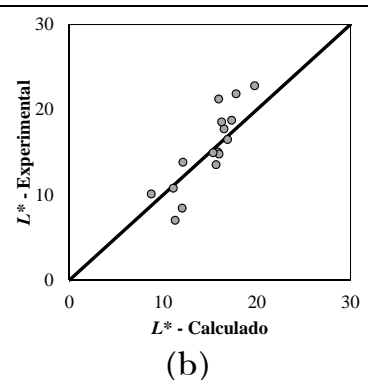

(b)
Figura 4 - (a) Relação entre $\varphi_{1}$ e $F r^{*}$; (b) Relação entre valores medidos e calculados de $L^{*}$

Equações 16 e 17

O uso da equação 16 resultou um coeficiente de correlação entre valores medidos e calculados igual a 0,86, próximo ao anterior. A soma dos erros quadráticos entre valores medidos e calculados passou de 105,5 (Equação $13 \mathrm{~b}$ com $\varphi_{1}\left(\mathrm{Fr}^{*}\right)$ ) para 97,8 (equação 16). Os coeficientes obtidos foram: $a_{0}=$ 0,$667 ; b_{0}=-2,06 ; a_{1}=1,46 ; b_{1}=-1,74$. Os resultados obtidos com a equação 17 foram inferiores aos obtidos com a equação 16 , com coeficiente de correlação igual a 0,75 e erros maiores, como pode ser visto na figura 5 . 


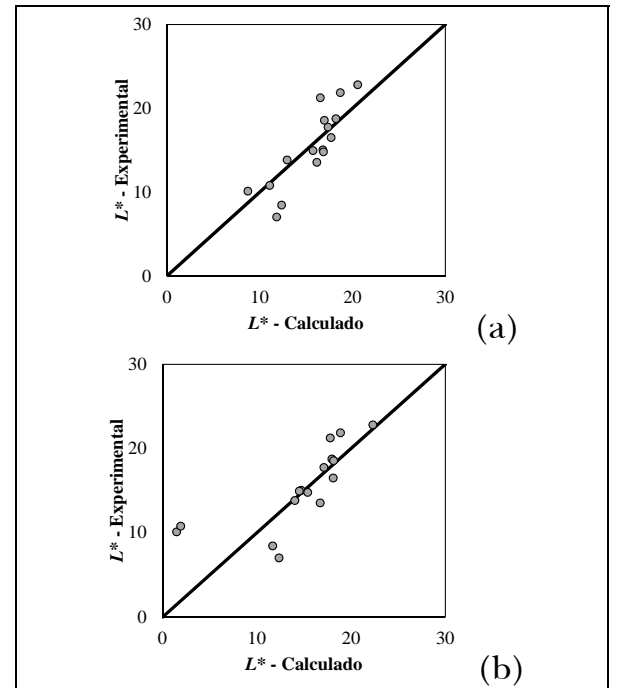

Figura 5 - Comparação entre valores medidos e calculados para $L^{*}$ : (a) Equação 16 e (b) Equação 17

Equação 18

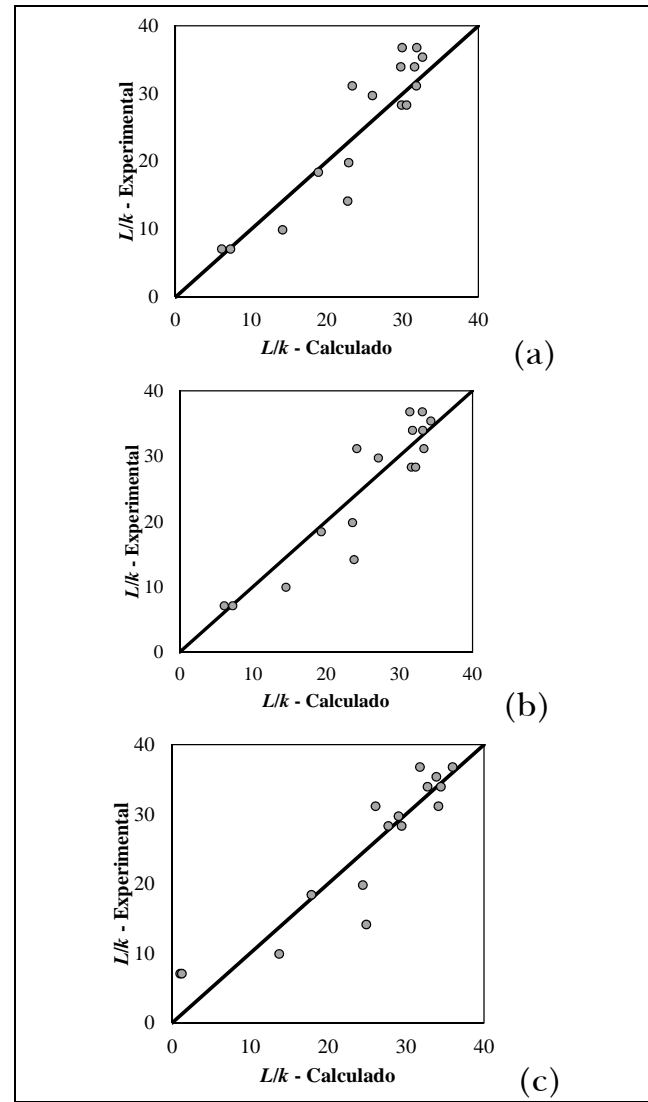

Figura 6 - (a) $L / k$ calculado com a relação entre $\varphi_{1}$ e $F r^{*}$ (Figura 4a); (b) Relação obtida com o uso das equações 14 e 18; (c) Relação obtida com as equações 15 e 18
O uso da equação 18 com a mesma função de ajuste $\varphi_{1}\left(F r^{*}\right)$, com $\mathrm{R}^{2}=0,97$, da figura $4 \mathrm{a}$, resultou em coeficientes de correlação maiores entre valores medidos e calculados. O coeficiente de correlação entre valores medidos e calculados para $L / k$ resultou igual a 0,92 . O gráfico desses valores (medidos e calculados) pode ser visto na figura 6a. As demais alternativas com as séries 14 e 15 e a equação 18 resultaram em coeficientes de correlação iguais a 0,92 também.

\section{CONCLUSÕES}

O presente estudo é original em toda a sua extensão, apresentando uma forma de abordagem para entender a interface de escoamentos bifásicos, nesse caso a água e o ar, como uma relação entre a taxa de geração de vazios na água, a vazão específica da água e o gradiente da profundidade da água na direção do escoamento.

Tal relação mostra-se fisicamente coerente, tendo-se demonstrado que a forma genérica da interface é explicada através da equação fornecida. Adicionalmente, ela permitiu apresentar previsões para o comprimento de transição entre duas condições de mistura do escoamento bifásico (nesse caso, entre água pura e mistura completa), em um escoamento particular ocorrendo em canais escalonados.

A equação básica segue as formulações de proporcionalidade entre taxas de transferência e gradientes de propriedades físicas comuns em fenômenos de transporte e em outros campos que envolvem movimento "difusivo". Nesse caso, a taxa de geração de vazios corresponde a uma taxa de transferência de ar através da interface.

O coeficiente de proporcionalidade foi motivo de maior discussão, apresentando-se soluções para o comprimento de transição considerando-o: 1) constante, 2) dependente da vazão específica e da profundidade do escoamento (esta última sendo uma variável de integração), 3) dependente apenas da vazão e da rugosidade (fatores constantes no problema matemático). Demonstrou-se que a taxa de geração de vazios pode variar com a posição $(x)$, sem implicar em alterações nas conclusões aqui apresentadas.

Em termos de comparação entre dados e previsões, a proposta (1) gerou uma correlação aceitável (coeficiente de correlação de 0,73 ), enquanto que a proposta (2) gerou um coeficiente de correla- 
ção bem melhor $(0,90)$ quando a solução rigorosa foi adotada (dois coeficientes de ajuste). Utilizando uma solução com coeficientes relaxados (cinco coeficientes de ajuste), a solução gerou um coeficiente de correlação de 0,98 . A proposta (3) foi multifacetada, produzindo também coeficientes de correlação entre 0,75 e 0,92 , utilizando ou uma função de ajuste entre o coeficiente de proporcionalidade e o número de Froude, ou duas propostas de série envolvendo duas e três constantes de ajuste.

Independente da aproximação utilizada para a evolução do coeficiente de proporcionalidade, verificou-se que se atingem bons resultados para a avaliação do comprimento de transição. Assim, entende-se que a presente formulação é adequada para quantificar os fenômenos vinculados à descrição da superfície livre em escoamentos aerados. A decisão sobre a melhor adimensionalização ou representação da constante de proporcionalidade depende de mais experimentos, a serem realizados com novas condições de declividade do canal, envolvendo a presença ou não de degraus, novas dimensões variáveis (geometria dos degraus, por exemplo), entre outros parâmetros que podem ser modificados.

Em termos de metodologia, vale mencionar que o presente estudo é resultado da convergência de diferentes questionamentos e propostas que foram discutidos entre os autores, podendo ser caracterizado como uma conversa contínua em clima de "tempestade cerebral" (brainstorming), onde sucessivas abordagens foram geradas e testadas. Eventualmente esse tipo de interação pode ser mais explorado em estudos específicos futuros.

\section{AGRADECIMENTOS}

Os autores agradecem ao $\mathrm{CNPq}$, pelo processo 141078/2009-0, a CAPES pelo processo 2201/06-2.

\section{REFERÊNCIAS}

ARANTES, E. J. Caracterização do escoamento sobre vertedores em degraus via CFD. 2007. $204 \mathrm{f}$. Tese (Doutorado) - Escola de Engenharia de São Carlos, Universidade de São Paulo, São Carlos, 2007.
BRITO, R. J. R. Análise da aeração em escoamentos de altas velocidades em calhas de vertedores. 2011. Dissertação (Mestrado) - Escola de Engenharia de São Carlos, Universidade de São Paulo, São Carlos, 2011.

CAIN, P.; WOOD, I. R. Instrumentation for aerated flow on spillways. Journal of Hydraulic Engineering, v. 107, n. HY11, 1407-1424, 1981.

CARVALHO, P. D. Aeração de escoamentos de alta velocidade em canais de forte declividade. 1997. Tese (Doutorado) - Escola de Engenharia de São Carlos, Universidade de São Paulo, São Carlos, Brasil, 1997.

CHOW, V. T. Open-channel hydraulics. New York: McGraw Hill Book, 1959. p.226-227.

KELLER, R. J., LAI, K. K.; WOOD, I. R. Developing Region in Self-Aerated Flows. Journal of Hydraulic Division, v. 100, n. HY4, p. 553-568, 1974.

LIMA, A. C. M. Caracterização de estrutura turbulenta em escoamentos aerados em canal de forte declividade com auxílio de velocimetria a laser. 2003. Tese (Doutorado) - Escola de Engenharia de São Carlos, Universidade de São Paulo, São Carlos, 2003.

LIMA, A. C. M.; SCHULZ, H. E.; GULLIVER, J. S. Air uptake along the lower nappe of a spillway aerator. Journal of Hydraulic Research, v. 46, n. 6, p. 839843, 2008.

LUEKER, M. L.; MOHSENI, O.; GULLIVER, J. S.; SCHULZ, H. E.; CHRISTOPHER, R. A. The physical model study of the folsom dam auxiliary spillway system. Minnesota: University of Minnesota, St. Anthony Falls Lab., 2008. (Project report, 511).

PETERKA, A. J. The effect of entrained air on cavitation pitting. Minneapolis, Minnesota: IAHR/ASCE, 1953. Joint Meeting Paper.

SCHULZ, H. E. Desenvolvimento de modelo teórico para a absorção de ar em aeradores de fundo. Relatório Final Pesquisa no Exterior (Report), Proc. CAPES BEX2201/06-2. Minnesota, U.S.A.: St. Anthony Falls Lab., Dept. Civil Engg. Univ. Minnesota, U.S.A., 2008. p. 94-100.

SCHULZ, H. E. O essencial em fenômenos de transporte. São Paulo: EDUSP, 2003. 382 p.

SCHULZ, H. E.; LOBOSCO, R. J.; SIMÕES, A. L. A. Multiphase analysis of entrained air in skimming 
flows along stepped chutes. In: ACOMEN, Int. Conf. on Advanced Computational Methods in Engineering, 5., 2011, Liège, University Liège. Proceedings... Liège: University Liège , 2011. p. 1-10.

SIMÕES, A. L. A. Considerações sobre a hidráulica de vertedores em degraus: metodologias adimensionais para pré-dimensionamento. 2008. Dissertação (Mestrado) - Escola de Engenharia de São Carlos, Universidade de São Paulo, São Carlos, 2008.

SIMÕES, A. L. A. Escoamentos em canais e vertedores com o fundo em degraus: desenvolvimentos experimentais, teóricos e numéricos. 2011. 157 f. Tese (Doutorado) - Escola de Engenharia de São Carlos, Universidade de São Paulo, São Carlos, 2011.

SIMÕES, A. L. A.; SCHULZ, H. E.; PORTO, R. M. Stepped and smooth spillways: resistance effects on stilling basin lengths. Journal of Hydraulic Research, v.48, n.3, p. 329-337, 2010.

STRAUB, L. G.; ANDERSON, A. G. Experiments on self-aerated flow in open channels. Journal of the Hydraulics Division, v. 84, n. 7, p. 1-35, 1958.

WOOD, I. R.; ACKERS, P.; LOVELESS, J. General method for critical point on spillways. Journal of $H y$ draulic Engineering, v. 109, n. 2, p. 308-312, 1983.

\section{Development Of The Free Surface In Air-Water Flows: Analogy With Basic Transfer Laws}

\section{ABSTRACT}

The interface in two-phase turbulent flows can be difficult to define, making it also difficult to quantify the relevant properties related to this interface. In the case of air-water flows, the large difference between the densities implies that, in many practical applications, the system is stratified, with a higher percentage of water in the lower region and a higher percentage of air in the upper region. Additionally, the atmosphere can be considered free of water, while deep flows can be considered free of air. Under these conditions, the position of the upper and lower interfaces of the air-water mixture can be studied more objectively, and the present study concentrates on the position of the upper interface, that is, the contact between the mixture and the atmosphere. The physical conservation laws always lead to useful balances, but frequently they involve numerous adjustments to take into account the simplified details. The present study provides a formulation considering the observation of the phenomenon and its immediate interfering variables, a usual procedure in the field of Transport Phenomena and which produces physically consistent conclusions. The formulation is applied to calculate the distance between black and full white water in rectangular stepped chutes.

Keywords: Transport phenomena. Two-phase flows. Channel steps. 